\title{
SURGERY OF THE RHEUMATOID DISEASES
}

\author{
By John Bastow, M.S., F.R.C.S.
}

Hon. Consulting Orthopaedic Surgeon, Devizes, Malmesbury and Warminster Hospitals;"Hon. Assistant Orthopaedic Surgeon, Royal National Hospital for Rheumatic Diseases, Bath ; Consulting Orthopaedic Surgeon, Wilts. E.M.S. Hospitals

The scope of surgery in the treatment of the rheumatoid diseases is, of necessity, limited by the impossibility of putting a definite term to the arthritic process. The progress of these diseases through relapses and remissions makes a definite end point very difficult to foretell.

For this reason there has been a tendency to allow the disease to 'burn itself out' before the surgeon is called in to repair, as best he may, the damage that the arthritis and the effects of prolonged decubitus have inflicted on the patient's joints and periarticular tissues. This is a cardinal error as the successful management of a case of rheumatoid arthritis calls for the co-operation of the physician and orthopaedic surgeon right from the onset.

The progress of deformity resulting from the joint lesions is insidious but surprisingly rapid in its development and its prevention calls for the application of protective splintage from the earliest stage of the illness. Such disabling conditions as ulnar deviation of the fingers, dropped foot with contracted tendo-achilles and triple subluxation of the knees may be seen in an untreated case within a few weeks of the onset of the disease and are extremely difficult to correct.

It is no use saying that the deformity which occurs during the acute stage can be corrected later on when the disease has become quiescent; as by that time a considerable amount of irreparable damage has been done.

In no other condition is the old saying that 'prevention is better than cure' of such vital importance. Of what use is it to arrest the disease by skilful medical care, gold injections, sanatorium régime, etc., if in the process of cure the patient is so hopelessly deformed as to be ever after severely handicapped in getting about, let alone being prevented from competing with his fellows in earning a living.

The surgical approach to the management of a case of rheumatoid disease may be divided into four phases. These phases in order of occurrence are :-

I. Application of protective splintage.

2. Surgical measures designed to arrest the local arthritic process.

3. Measures designed to correct established deformity.

4. Reconstructive surgery.

To consider these phases in detail :-

\section{Application of protective splintage during the acute stage}

The role of the surgeon in this stage of the disease is not spectacular but it is of the utmost importance. He must insist that the most vulnerable joints, i.e. the knees, ankles, wrist and fingers are protected by light, carefullymoulded splints made of plaster, poroplastic or plastic materials, so that they are maintained in the position of optimum function.

The position of optimum function for arthritic joints is now fairly well standardized and varies within narrow limits, depending to some extent on the patient's occupation and the consequent demands on each particular joint should ankylosis occur in the rest position.

The following table is taken from an article on Physiological Rest by Mr. N. Capener in the British Medical Fournal, November 23, 1946. 
Table showing the Optimal Positions for the Treatment of Arthritic Foints

\begin{tabular}{lll}
\hline Joint & Position & Remarks \\
\hline
\end{tabular}

Shoulder

Abduction $45^{\circ}$

Flexion $30^{\circ}$

External rotation $15^{\circ}$

Elbow Extended to about $100^{\circ}$

Forearm Mid prone supine position

Wrist $\quad$ Extension $30^{\circ}$

Digits and thumb

Prevents stiffness in adduction; aids deltoid function; safeguards capsular integrity. The position is favourable for function in ankylosis.

Safeguards the function of the elbow flexor muscles. If ankylosis is likely to occur, modifications of the position recommended must be made according to the wishes of the patient, guided by his social circumstances and occupation. Where both elbows are involved, one should be treated at a smaller angle.

Favours the restoration of either movement. If ankylosis occurs compensation for loss of pronation can be gained by abduction of the shoulder. Stiffness in supination is objectionable.

The angle is measured upon the dorsum of the radius and of the third metacarpal.

The hand as a unit is held in the position of semi grasp, the thumb practically continuing the line of the lateral border of the radius, the phalanges of all digits in the position of natural relaxation, e.g. that assumed when the normal hand is loose at the side of the body. Capsular and ligamentous stretching is avoided. The vicious position of metacarpo-phalangeal hyperextension is avoided.

Spine Normal curves maintained

Hip

Abduction only of sufficient amount to compensate for true shortening

Rotation nil

Flexion $20^{\circ}$

Knee $\quad$ Flexion $15^{\circ}$

Ankle Right angle

Tarsal joints

Neutral as to inversion or eversion

Toes

Maintain flexion of M.T.P. joints and extension of I.P. joints

Flexion as well as lordosis of the lumbar spine to be avoided.

In arthritis extreme degrees of true shortening of a lower extremity are unlikely to be found. Flexion permits sitting if there is compensatory movement in the lumbar spine.

Avoids posterior capsular stretching and resultant hyperextension instability. The competence of the quadriceps is adequately safeguarded.

If ankylosis appears inevitable the foot should be plantar flexed $5^{\circ}$ to $10^{\circ}$ in men and a few degrees more in women to facilitate walking in high heel shoes.

While the arches of the foot should be preserved, it is better, should ankylosis be inevitable, to err in the direction of eversion, for the weight-bearing surface exposed to the ground is less likely to be troublesome than if the foot is inverted or cavoid.

Preserves intrinsic muscular activity; avoids metatarso-phalangeal subluxation and metatarsal pressure points. 
It has been my experience that a position just short of full extension can be allowed in the knee joint with safeguards, to be mentioned in the next paragraph, but otherwise these positions may be accepted as standards.

It is, however, important that each joint should be actively moved through its full range of movement once daily to prevent the development of adhesions, and this applies with equal force to the joints that are not splinted, such as shoulders, elbows and hips.

The bed must be firm and flat and care taken that the patient is not forced by a nest of pillows into a turtle backed position that may result in a permanent kyphosis with flattening of the chest, visceroptosis and its attendant evils.

The provision of an overhead beam on the patient's bed with slings and pulleys to assist in movement by eliminating the effects of gravity is a great help now, as at all stages of the disease.

Above all, the swollen, tender, painful joints must not be subjected to the trauma of constant use. There is no quicker way of permanently damaging an inflamed joint in the lower limb than by walking about on it, and a joint in the upper limb than by keeping it in constant use.

However, all too often the surgeon is called in when some degree of deformity already exists and then more active measures are called for, which will be considered later under heading 3 .

Technique of splintage: The material chiefly used for the application of protective splintage in arthritis has hitherto been plaster of Paris. This is a very satisfactory material to use, provided care is taken in its application to avoid any risk of the formation of pressure sores.

The plaster splints must be light, very carefully moulded to the contours of the limb and can, with advantage, be applied over a single layer of stockinette.

It is essential that during the application of these plasters, no encircling turns of bandage should be pulled tight, each layer of plaster must be carefully massaged into the next and great care must be taken that the edges of the splints are smooth and do not cause chafing.

Many plaster splints are spoilt either by their being moved before the plaster is set, which causes the plaster to crack, or by resting them on hard surfaces such as table tops so that indentations are made in the plaster which are liable to cause pressure sores.

After the plasters have been bivalved, that is if they have been originally made as complete plasters, and this is often advisable in the lower limb for ensuring accuracy in fitting, the inside of the plaster can be cellulosed or varnished to avoid deterioration due to contamination by sweating.

Joints such as those of the feet, ankles, knees and spine, and those of the fingers, wrists and elbows, are suitable for plaster fixation. The shoulder is usually immobilized by means of a modification of the Littler-Jones abduction splint or, when the patient is confined to bed, by suspension of the arm, and forearm from slings suspended from an overhead beam.

It is seldom wise, however, to encase the fingers completely in plaster and a splint to support the hand and fingers should be made as a moulded slab on the palmar aspect only.

\section{Surgical measures designed to assist in arresting the local arthritic process}

The chief indications for surgery on this account in the acute stage of arthritis are :-

(a) The persistence of joint inflammation and effusion in spite of rest, splintage and the usual physical and medical methods of treatment.

(b) Persistent pain in the joint unrelieved by splintage, rest and medical measures.

It often happens in a case of rheumatoid arthritis that one or two of the large joints, typically the knees, remain hot, swollen and painful in spite of treatment, and here the surgeon may properly consider whether local operative measures may be undertaken in the hope of relieving the local condition.

General considerations for operation on arthritic joints. Certain precautions should be taken in all cases where arthritic joints are subjected to operative procedures to avoid the troublesome complications of haemorrhage and infection that may otherwise turn a successful operation into a surgical catastrophe. 
Firstly, each operation must be performed under the strictest aseptic technique and preferably in a clean theatre where no infected cases are handled. The optimum is an orthopaedic theatre reserved for bone and joint cases.

Secondly, all cases should be operated upon under penicillin cover, i.e. large doses of penicillin such as 200,000 units eight hourly should be given intramuscularly, beginning when the patient is under the anaesthetic, and continuing for four days after operation.

Thirdly, where possible the operation should be performed under a tourniquet, preferably pneumatic, after the limb has been previously exsanguinated by means of an Esmach's bandage.

Fourthly, a copious pressure dressing should be applied to the joint for the first 24 hours after operation.

The first thing to be considered is, the simple but useful little operation known as joint lavage.

Operative technique. Taking the knee joint as the one where this measure is most often called for, the joint is exposed by a small incision in the suprapatellar pouch, extending obliquely upwards and outwards from the superior angle of the patella-either on the outer or inner side-and splitting the vastus internus or externus muscle in the line of its fibres.

The interior of the joint is inspected, the state of the articular cartilage covering the patella and upper part of the femoral condyle is noted, and a soft, rubber catheter is inserted into the joint cavity.

The joint is then thoroughly washed out with two pints of a warm saline or dilute Eusol solution by means of a funnel and tube, all corners of the joint are carefully flushed out, filmy adhesions are broken and a varying amount of fibrinous debris, looking like coagulated egg white is expressed. During the lavage, the joint is gently flexed and extended to ensure that the posterior pouches of the capsule are filled with the fluid.

Finally, the catheter is withdrawn, the small wound closed in layers, a pressure bandage is applied over wool, the tourniquet removed and the split rest plaster re-applied.

Post-operative routine. After the pressure dressing is removed, gentle active contractions of the overlying muscles are encouraged and after four days, the limb is lifted out of the splint once daily for assisted active movements, preferably with the limb supported in slings from an overhead beam to assist movement by eliminating the effect of gravity. "After ten days, the sutures are removed and the usual routine of treatment for arthritis such as baths, physiotherapy, etc., can be proceeded with.

It is surprising how often this simple procedure is effective in securing the resolution of the local inflammatory (or alergic) signs and symptoms and causing the joint to become cool and painless.

Synovectomy. In cases where the joint effusion is of more long standing one finds on operation a chronically thickened and congested, or sometimes a pallid and ' wash leather' appearance of the synovia, often with a fringe of swollen, hypertrophied villi round the articular margin.

An irreversible process appears to have been set up in the joint tissues and the only remedy which holds out any hope of arresting the condition is to perform a synovectomy, removing as much as possible of the diseased joint lining and relying on metaplasia of the remaining connective tissue to form a new joint cavity.

Now this operation requires a most careful selection of cases if successful results are to be achieved.

(a) Firstly there is the patient's morale to consider and here it must be stressed that no major surgical procedures must be carried out on arthritic patients unless there is a reasonably good prospect of the patient co-operating in the necessary after treatment. Early joint movements after synovectomy are essential to a successful result and must be persisted with in spite of a certain amount of pain. Unless the patient possesses the will to recovery and is willing to persevere in regaining the use of the joint the surgeon's efforts will be largely wasted.

(b) Age of patient. The younger the patient, the better the chance of success, but age is no bar to the performance of the operation unless there is accompanying gross osteoarthritic change in the joint concerned.

(c) Type of joint reaction. The ideal cases for operation are those with a greatly distended 
joint cavity, either as the result of recurrent effusion of synovial fluid or by the accumulation of hypertrophic svnovial villi.

(d) State of joint surfaces. Better results are obtained if $\mathrm{X}$-ray shows that a fair joint space exists, i.e. the articular cartilage has not vet been eroded away and no marked secondary osteoarthritic changes have supervened.

(e) The muscles must be capable of moderate development so that movements of the joint can be maintained. If the muscles are so wasted and fibrotic from long-standing disuse that no regeneration is likely, the operation should not be performed.

Operative technique. The knee may be taken as the most usual joint to be operated upon. A long antero-medial patellar displacing incision is usual and the suprapatellar pouch is dissected out in its entirety with the lateral prolongation of the synovial sac under the lateral ligaments, taking care to preserve the latter intact. Then the infrapatellar pad is explored and all thickened synovia removed, including the alar folds and the reflection of synovia over the cruciate ligaments. The posterior synovial pouch of the knee is not opened.

The wound is then closed in layers and a small rubber drain inserted into the suprapatellar space. An ample pressure bandage is applied over wool and a split plaster is applied, reaching from toes to groin. Quadriceps exercises are encouraged from the start.

After 48 hours the pressure dressing and the rubber drain are removed and after four days, gentle active movements of the knee are begun daily. After ten days the sutures are removed and the patient begins exercises with the limb supported by slings and pulleys. He can also, at this stage, begin movements in an immersion bath.

After three weeks, if the range of movement is small and shows no sign of increasing, the knee is flexed to $90^{\circ}$ under pentothal anaesthesia. Numerous adhesions can be felt to give way with this manoeuvre and the range of movement is immediately increased. Walking can be commenced with a splint behind the knee during the third week, and a second manipulation can, if necessary, be carried out in the sixth week.

About $90^{\circ}$ of movement, and more in favourable cases, can be expected but, occasionally, if the quadriceps is already very contracted, only $30^{\circ}$ can be obtained. However, in nearly all cases, a cool and painless joint can be obtained and this is well worth some sacrifice of movement.

\section{Case Records}

To illustrate synovectomy

Mrs. R. Aged 53 years. Laundress.

21.2.46 Admitted to Royal National Hospital for Rheumatic Diseases.

History Pain and swelling of left knee of four years' duration. Recurrent effusion that had been aspirated many times. Three months previously the right knee had begun to swell and become painful. Corrected Sedimentation Rate -62 per cent.

B.U.A.-3.40 migs. per 100 c.c. Berger-negative.

Treatment Rest plasters applied. Medical treatment. Physiotherapy instituted.

Progress Swelling of right knee subsided under treatment, but left knee remained troublesome and swollen.

Aspiration Fluid report.

Appearance-turbid greenish yellow.

Protein 2 per cent.

Mucin-positive.

Cells- 3.400 c.mm.

Polymorphs-17 per cent.

Lymphocytes - 45 per cent.

Monocytes-10 per cent.

Plasma cells -4 per cent.

Synovial cells-24 per cent.

Gram-no organisms seen.

Ziehl Neelsen-no acid fat bacili seen.

Culture-sterile.

The fluid shows the úsual characters of that of rheumatoid arthritis. The cell count is rather lower than usual and lymphocytes predominate, but these proportions may be found in rheumatoid arthritis.

4.4.46 Synovectomy performed on left knee. Through a 5 in. incision along medial side of patella and quadriceps tendon, patella reflected and joint capsule dissected away from synovial membrane. Whole of synovia of suprapatellar pouch and front side of joint dissected out and reviewed. Hypertrophy. '. Numerous villous processes on inner surface of synovia. Joint surface of femur partly destroyed around edges. Joint capsule and subcutaneous tissue closed in layers. P.O.P. applied. 
28.5.46 Left knee moves $175^{\circ}$ to $90^{\circ}$. No effusion. Discharged from hospital and physiotherapy continued at an outpatient clinic. Walks well.

$\mathrm{X}$-ray-see Figs. I $\mathrm{a}$ and $\mathrm{I} b$.

Comment. This case illustrates one type of case that responds well to synovectomy where villous hypertrophy of the synovia predominates in one joint. This case may well have progressed to a general arthritis of rheumatoid type, and it is considered in such cases that the surgical arrest of a predominant joint lesion may assist in preventing further arthritic lesions from developing elsewhere.

Mr. H.R.S. Aged 25 years. Clerk.

29.3.46 Admitted to Royal National Hospital for Rheumatic Diseases, with a three years' history of recurrent synovitis of both knees.

In September, 1945, patient was discharged from hospital as a case of 'idiopathic' synovitis, all investigations as to possible Wassermann infection, Tb., etc., having been proved negative. Left knee eventually settled down but right knee remained swollen and weak.

On admission. Right knee swollen.

C.S.R. - 93 per cent.

B.U.A.- 3.6 mgms. per .100 c.cs.

Berger-negative.

$\mathrm{X}$-ray-joint surfaces in good condition.

16.4.46 Synovectomy performed on right knee. Pathologist's report on synovia'typical changes of rheumatoid arthritis.'

\section{Progress}

$14 \cdot 5 \cdot 46$

Knee manipulated to increase range of flexion.

17.5.46 Discharged from hospital.

Knee moves $180^{\circ}$ to $110^{\circ}$.

In 1947 Patient reports :- Knee has full range of movement. No pain or swelling. Is in full work and has been so for six months.

$\mathrm{X}$-ray-see Figs. 2a and $2 \mathrm{~b}$.

Comment. This case illustrates a good response to synovectomy in a young patient and in view of pathologist's report, it is considered that if synovectomy had not been performed, a progressive deterioration would have taken place in the condition of this joint.

Another procedure of value in selected cases of arthritis involving the knee joint is patellectomy.
Indications. Incidence of arthritic change is chiefly confined to the patello-femoral articulation.

Symptoms. I. Pain on active flexion and extensions of the joint felt by the patient as being ' under the knee cap.'

2. Pain when the patella is pressed against the underlying femoral condyles.

Signs. I. Subpatellar crepitus on movement.

2. X-ray appearances of subpatellar arthritis, especially :-

(a) Narrowing of patello-femoral joint space.

(b) Appearance of erosion of subpatellar articular surfaces.

(c) Relative good joint spacing in the tibio-femoral articular area, indicating that the weight bearing part of the articulation is still capable of being salvaged. See Figs. $3 \mathrm{a}$ and $3 \mathrm{~b}$.

Operative technique. Operation is performed with a tourniquet, the limb having been ensanguinated by an Esmach's bandage. A curved parapatellar incision is employed and the patella is removed through a horizontal incision in the aponeurosis of the quadriceps. The joint cavity is explored and if the synovial membrane is either grossly hypertrophical or looks very pale, fibrotic and thickened, a synovectomy is performed. Otherwise, any hypertrophied synovial fringes are excised and the synovial membrane left intact. The wound is then closed by thread or fine stainless steel sutures, the quadriceps tendons being firmly bunched together over the front of the joint. The skin is closed, a pressure dressing is applied and a split plaster in full extension (which has previously been prepared) is applied to the limb after removal of the tourniquet.

After treatment. The pressure dressing is removed after 48 hours and quadriceps contractions are encouraged from the start. After ten days, the sutures are removed and gentle active exercises of the joint are begun. It is helpful at this stage if the limb can be exercised in a warm bath or else supported by counter-weighted slings from a Balkan beam, 
to eliminate the effects of gravity. In general, the rate of progress is slower than in a patellectomy following a fracture, but walking with a back slab on the knee can be allowed in the third week.

After six weeks, the back slab can be dispensed with and walking allowed with the support of a crepe bandage only. The chief aim of after treatment is to secure good recovery of tone in the quadriceps. It is most important to keep the power of full extension of the joint and flexion must not be forced at the expense of extension. Unless the patient regains the power to lock the joint in full extension, then the knee is apt to remain weak and tends to 'let him down.'

Complications-Haemarthrosis. This is sometimes met with following synovectomy and must be dealt with by aspiration of the joint and the re-application of a pressure bandage. Progress will be delayed if a haemarthrosis occurs, but it does not preclude a successful result.

\section{Case Records}

To illustrate synovectomy plus patellectomy

\section{Mr. T.P. Aged 62 years. Miner.}

History 14 months pain and swelling in wrist, finger joints, knees and ankles, with a sudden onset.

13.3.42 Admitted to Royal National Hospital for Rheumatic Diseases.

Diagnosed as active rheumatoid arthritis.

Corrected sedimentation rate-62 per cent.

Treatment Rest, course of gold injections, baths and physiotherapy.

27.4.42 Discharged home to continue treatment.

21.5.43 Re-admitted to hospital. Now complains chiefly of painful, swollen knee joints, wrist and finger joints.

Treatment Rest plasters applied, baths and physiotherapy. Discharged.

26.II.43 Re-admitted to hospital. Knee joints still troublesome.

25.1 2.43 Joint lavage performed on both knees.

28.r.44 Discharged to home.

20.8.46 Re-admitted to hospital. Now complains of residual trouble in left knee. $\mathrm{X}$-ray shows secondary osteoarthritic changes in left knee.
O.E.-joint swollen and painful.

20.9.46 Synovectomy combined with patellectomy performed. Pathologist's report on synovial membrane :- ' The synovial membrane showed extensive fibrosis with replacement of large areas by a dense collagenous fibrous tissue. Some areas of lymphocytic infiltration were present. In addition there were patches of histrocytic reaction, these phagocytic cells being engorged with brown pigment, probably altered blood. They were associated with granulation tissue formation, probably organizing parts subjected to trauma. The villi were very fibrous and ulceration of the synovial covering had occurred in large areas. The appearances suggested old rheumatoid arthritis, the subject of secondary traumatic effects. Muscle ( $V$. medialis). Two areas of rather doubtful round cell reaction were noted (I) between fibres (2) near a large thick-walled artery. Slight degenerative changes in muscle were present.'

I I.I I.46 Discharged, wearing a removable plaster splint on the left knee and regaining a useful range of movement.

$\mathrm{X}$-ray-see Figs. $4 \mathrm{a}$ and $4 \mathrm{~b}$.

Comment. This case illustrates well the need for continuous supervision of these cases and methods for dealing with residual joint trouble by surgical means only after conservative methods have failed to give relief.

Bone drilling. 'T his is a useful measure for relief of pain in certain cases that has been greatly abused in the past. The indications are strictly limited :-

I. Persistent pain in a joint, especially if nocturnal, that is not abolished by rest, splintage and local heat, in the presence of :-

2. X-ray appearances of sclerosis in the subarticular bone.

X-ray. See Figs. $5^{a}$ and 5 b.

The assumption is that the pain in such cases is largely due to osteitis in the bones taking part in the joint, and that relief of tension in the bone achieved by drilling a hole through the cortex into the medulla will tend to relieve this. Hence the procedure would be chiefly of value in chronic cases where secondary osteo-arthritic changes have developed in a joint previously damaged by 

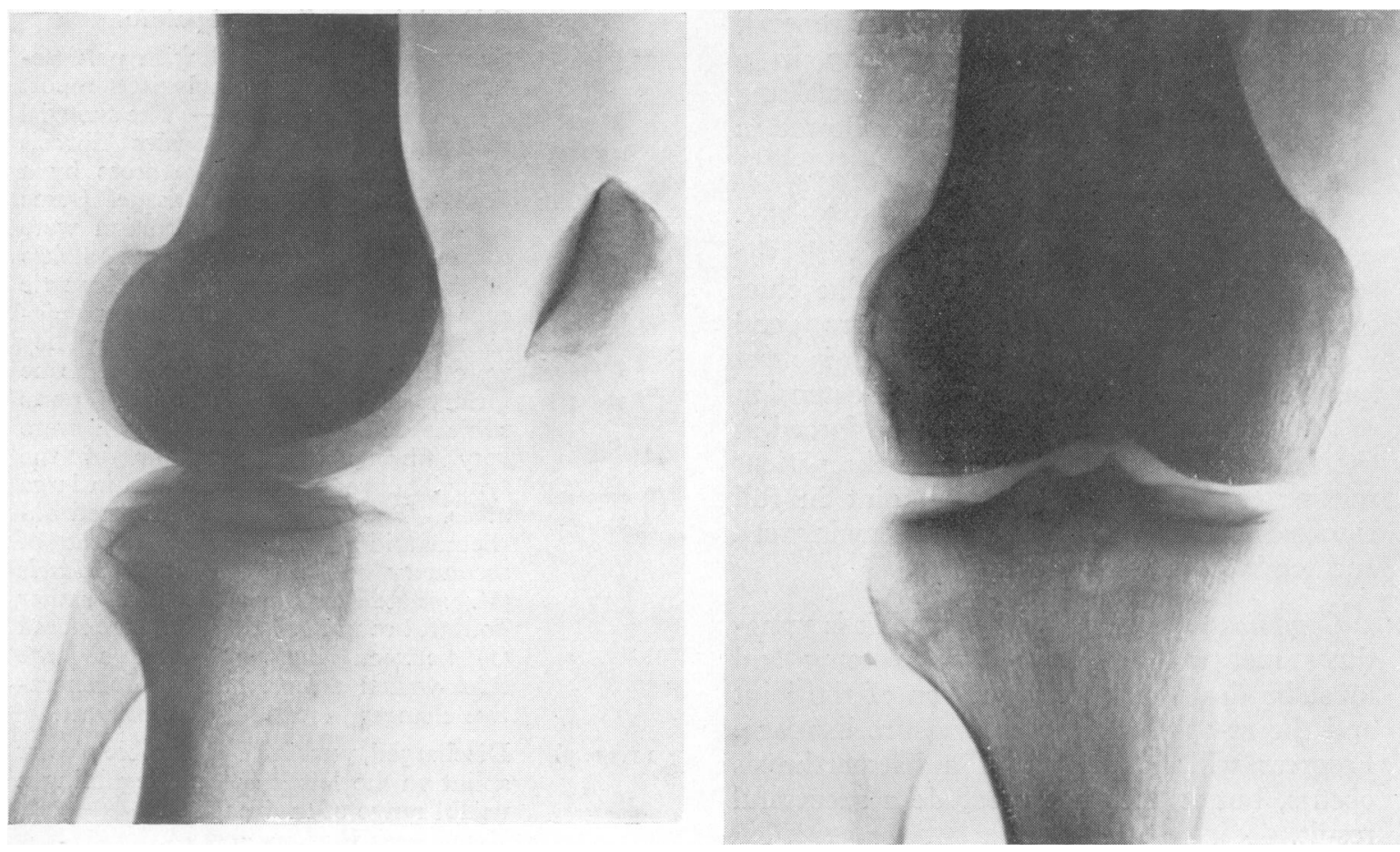

(a) Distrusion of joint space in lateral view

FIG. I

(b) Good joint spacing in A.P. view
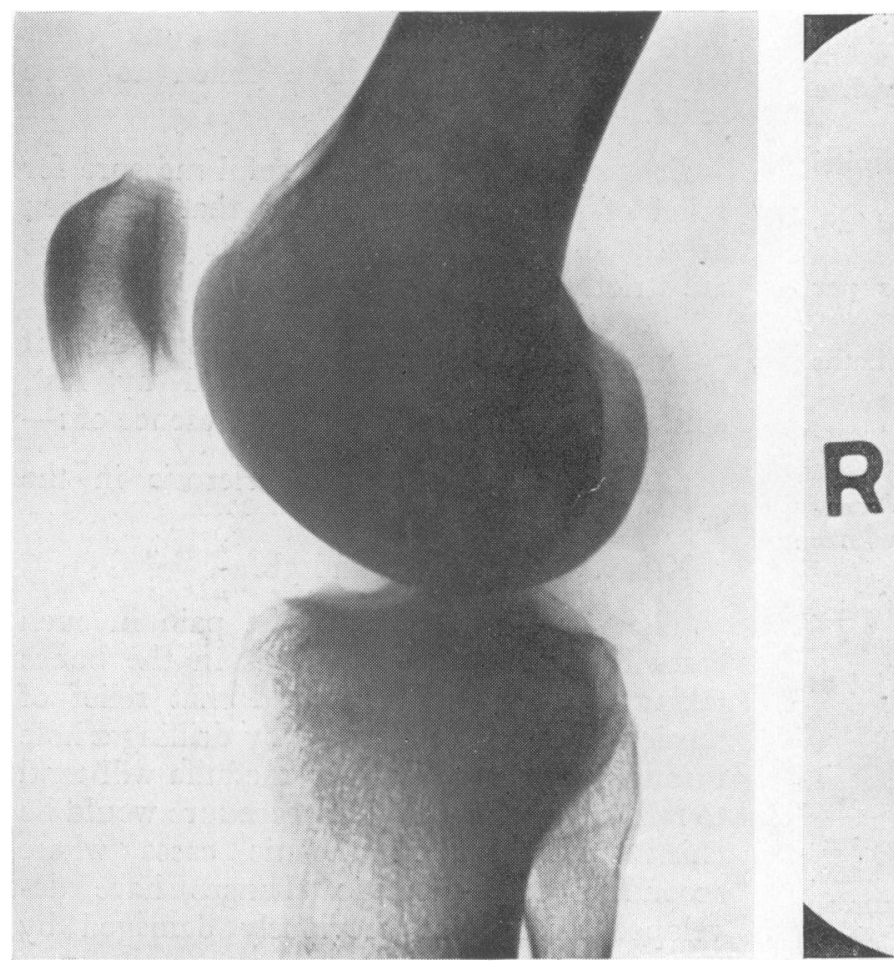

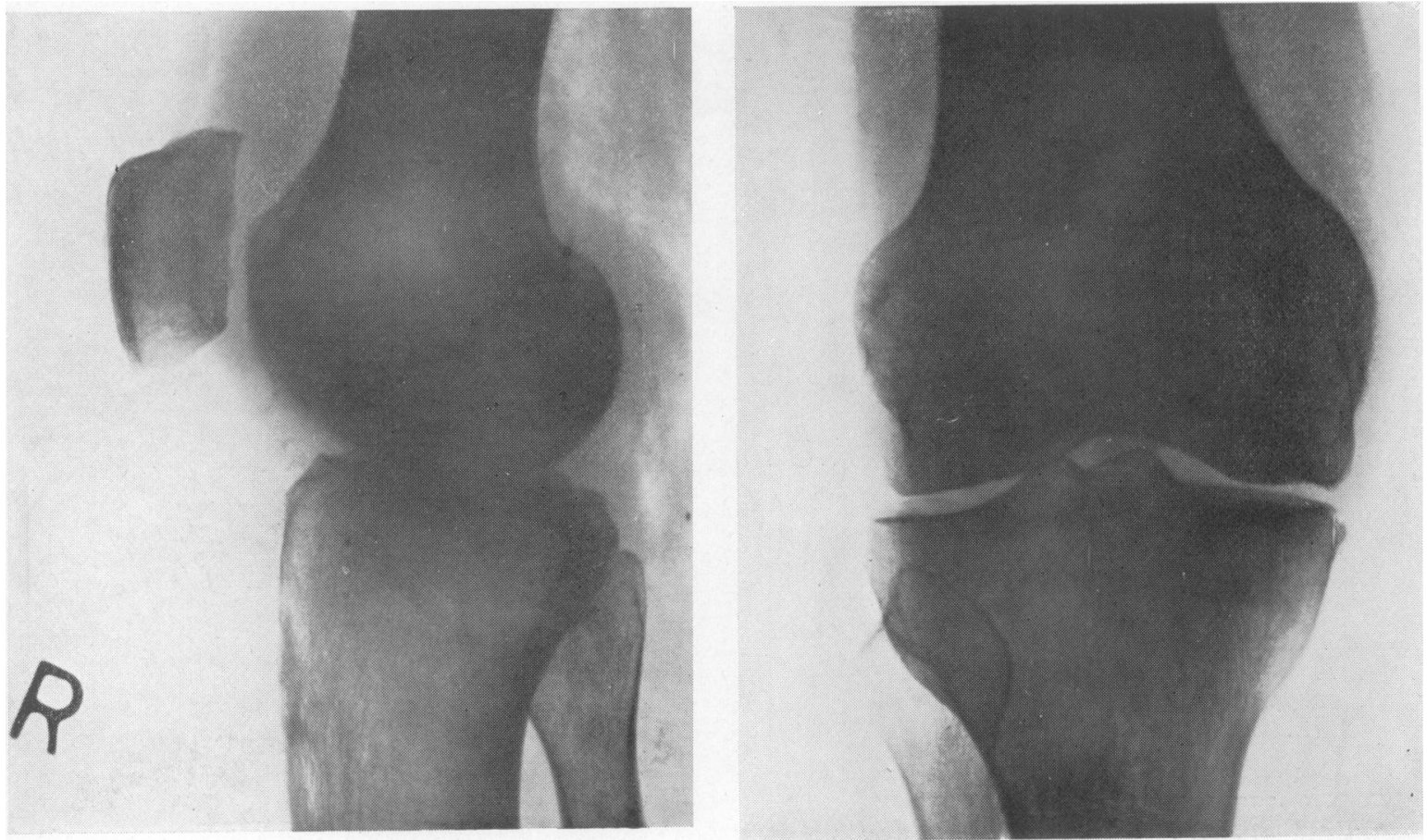

(a) Sub-patellar erosion

FIG. III

(b) Reasonably good condition of tibio-femoral weightbearing articular surface

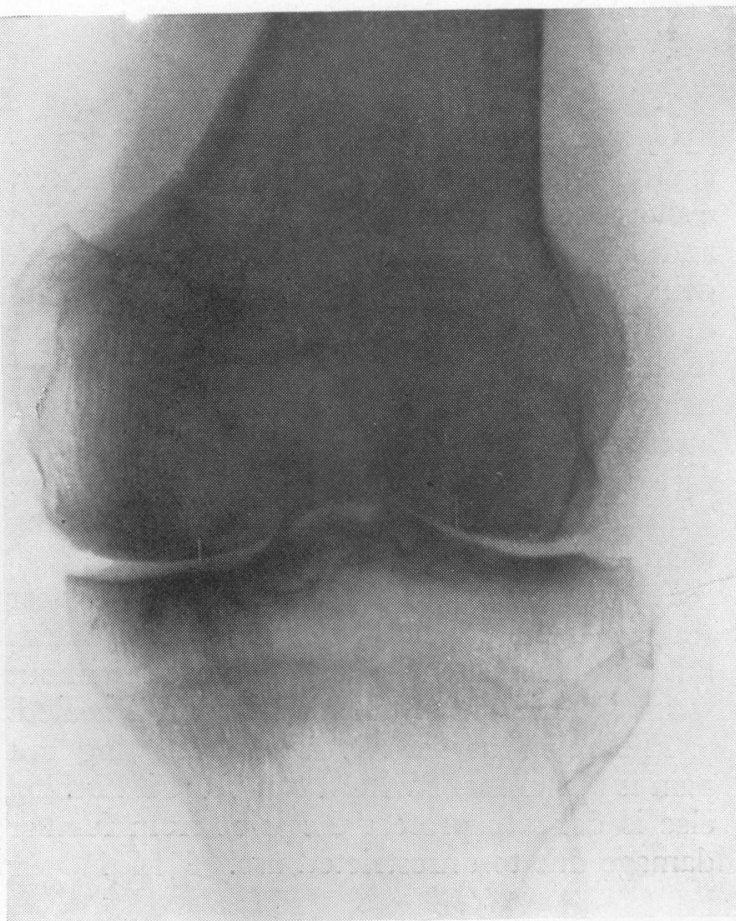

FIG. IV (b) Narrowed joint space and some erosion in A.P. view 

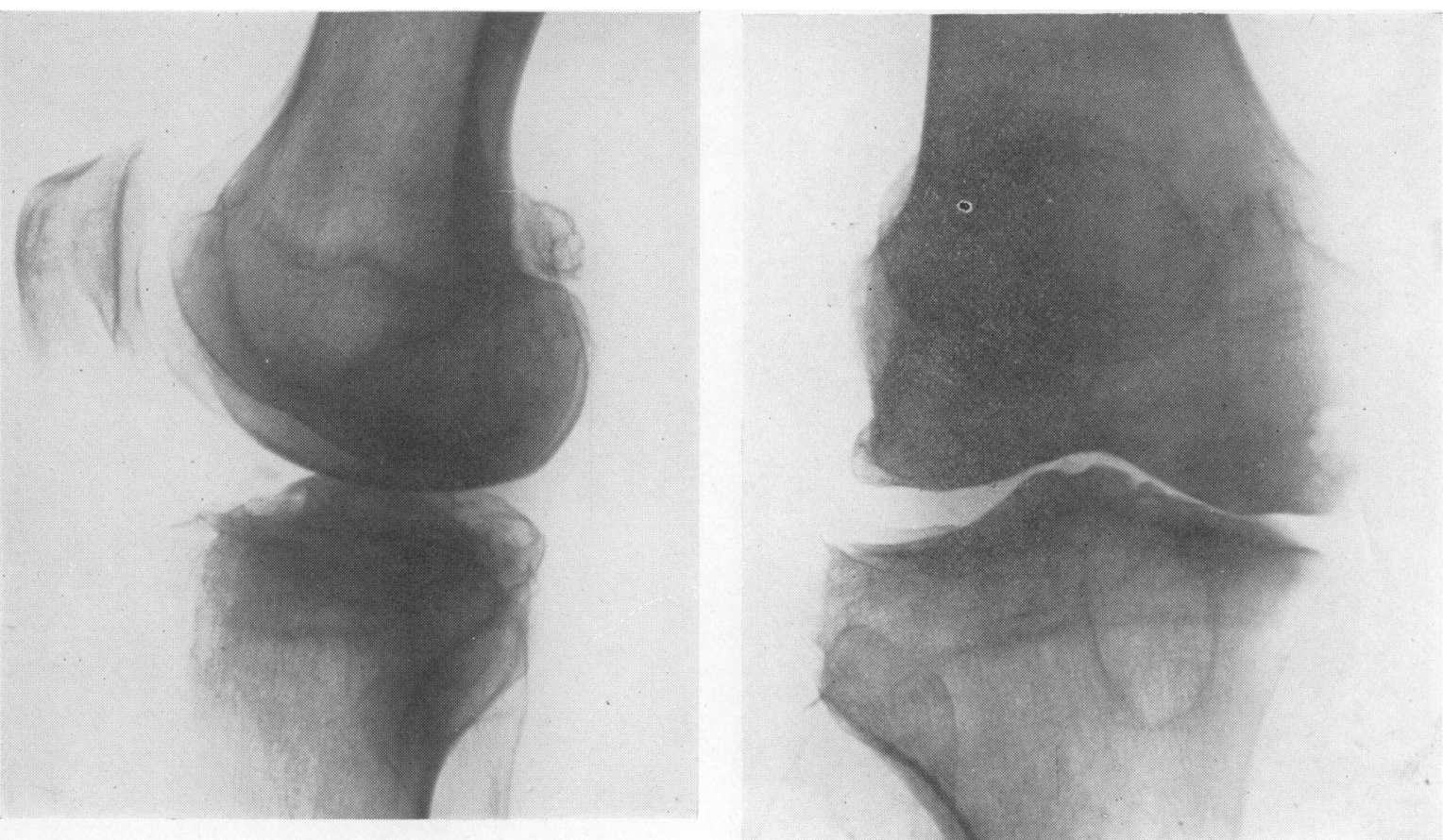

FIG. V

Sub-articular sclerosis, secondary osteoarthritic lipping and cystic changes in ends of bones-suitable for bone drilling

rheumatoid disease. Joints where this procedure is often helpful are :-

I. The hip joint.

2. The knee joint.

3. The wrist joint.

Operative technique. Under the usual precautions, i.e. strict asepsis and penicillin cover, a short incision is made over the bone involved and a hole is made in the cortex with a $\frac{1}{4}$ in. drill. The medulla is then cautiously drilled in several directions fanning out from the hole in the cortex towards the articular surface, but using a small drill so as not to destroy the internal architecture of the bone and taking care not to penetrate the articular cartilage. The skin only is closed and a pressure dressing is applied.

It is wise not to allow the patient to bear weight on the affected joint for several weeks, but free movement can be allowed without weight bearing as soon as the wound is healed.

The relief of pain is often gratifying but pain is apt to recur within two years if nothing else is done to protect the joint from further damage due to unrestricted use.
3. Measures designed to correct established deformity

One can distinguish four stages in the development of deformity in the arthritic joint :-

I. Deformity of recent onset due to muscle spasm alone. This muscle spasm can be abolished by full anaesthesia and the deformity controlled by the application of a rest plaster applied in the position for optimum function without any forcible correction being necessary. Any return of muscle spasm is dealt with by the exhibition of pain relieving and sedative drugs and the plasters can be removed temporarily after 24 to 48 hours to allow the usual daily movements to be undertaken and physiotherapy to be instituted. The splint must, however, be worn for the rest of the 24 hours when active treatment is not being undertaken, as it must be remembered that rest and not activity is the keynote in the treatment of an acute arthritic process.

2. Deformity which is not relieved completely by full anaesthesia and is due to the 
development of adhesions in the joint and periarticular structures and contracture of the muscles on the side of the deformity. Here carefully controlled but forcible pressure is needed to correct the deformity and immediately correction has been obtained, a carefully moulded plaster splint should be applied. It is wise to apply one layer of wool bandage to the joint in this case before applying the plaster, to allow room for reactionary joint swelling.

The post-operative routine is similar to that in the above category but more sedatization will be required and the plaster should not be removed for 48 hours in order to give the spasm a better chance to settle down.

3. Deformity which persists under anaesthesia and cannot be completely corrected by manipulation at one session.

One soon learns by experience how much force can be applied at one sitting to correct deformity without stirring up undue joint reaction and without risk of fracturing the bones taking part in the joint. It is always better to do too little than to attempt too much.

Post-operative routine will be similar to that of the previous case but one or more further manipulations will be required at intervals of two or three weeks before the optimum position can be maintained.

It is helpful to measure the range of movement in the joint weekly with an angle meter and continue with physiotherapy as long as the range of movement is increasing. As soon as progress comes to a standstill, then the time for the next manipulation has been reached.

If this regime is followed, then joint reaction will be minimal and there is no risk of a 'flare up.' of the local arthritic process. Such manipulations which are immediately followed by splintage can be undertaken at any time during the progress of the arthritis without risk.

It is the use of forcible unrestricted manipulations without the protection of splintage afterwards which gives such disastrous results in these cases.

In old-standing cases where the deformity is of long standing there is a good deal of fibrosis of the joint capsule and shortening of the surrounding muscles from contracture on the flexor aspect of the joint.' In such cases, although the deformity may be capable of correction by manipulation and splintage, the total range of movement of the joint is not likely to be increased, i.e. a flexed knee that moves $90^{\circ}$ to $120^{\circ}$ before treatment, may be expected to move $180^{\circ}$ to $150^{\circ}$ after correction and no more. It is always wise to warn the patient of this beforehand.

4. Deformity that requires surgical measures for its correction.

(a) Tenotomy. There are certain deformities such as adduction contracture of the hip, dorsal contracture of the extensor tendons of the toes, drop foot, due to contraction of the tendo-achilles, where it is necessary to tenotomize the contracted tendon before correction can be obtained.

Two methods are used :-

(i) Subcutaneous tenotomy, using a small tenotomy knife inserted through a puncture in the skin, is often successfully employed in cases of tenotomy of the tendons of the toes -both flexors and extensors or both as required-and in the adductor longus tendon of the hip.

(ii) Open tenotomy, as part of a formal operation where the tendon is elongated by means of an oblique or Z-shaped incision and then sutured together in an elongated position, is usually employed in the case of the tendo-achilles or in the case of contracted hamstring tendons in the flexed knee.

(b) Capsular slide operations, such as Souttar's procedure for the flexed hip or Walton's operation for the flexed knee, are more extensive procedures in which contracted muscles and capsular structures are slipped from their attachment to the bone by means of a sharp periosteal elevator and the flexed joint then straightened by manipulation. Here care must be taken to guard against endangering the circulation of the limb by stretching the contracted vessels too, quickly and also to ensure against stretch paralysis of nerves by stripping all affected nerves free from adherent tissues well above and below the centre of the wound.

(c) Bone operations. This includes all varieties of osteotomies and osteoclases, as set 
out in textbooks of orthopaedics and the procedures of arthrodesis, arthroplasty and pseudarthrosis, considered in a later section of this paper.

The place of manipulative treatment in the Rheumatic diseases.

I. It may be stated categorically that manipulation has no place in the acute stages of these diseases.

2. During a remission or in the quiescent phase of the disease, manipulation is often of definite value but the indications are strictly limited. Certain rules must be observed :-

(a) No joint must be manipulated without a preliminary $\mathrm{X}$-ray to exclude the presence of :-

(i) Bony ankylosis.

(ii). Tuberculous disease.

(iii) The presence of cancerous metastases in the bones involved.

(b) No joint must be manipulated in the presence of an intercurrent infection or an active inflammatory process elsewhere in the body.

(c) Such joints as the elbow or the interphalangeal joints of the fingers should never be manipulated under an anaesthetic.

(d) 'Little and often' is the safe rule. Attempts to gain too much movement at one session will nearly always defeat their own object by stirring up too much reaction and causing secondary joint stiffness to occur.

(e) As a general rule it is wise never to manipulate a joint with a view to increasing its range of movement if it is hot and swollen to the touch.

(f) Repeated gentle manipulations without anaesthesia are often very helpful in cases where many joints are involved and can well be carried out by a skilled physiotherapist. Such manipulations are best performed after muscle spasm has been relaxed by local heat or by immersion in warm saline or mineral baths or mud packs.

(g) In all cases of rheumatoid disease, the bones are decalcified and the decalcification is maximal in a zone corresponding to the metaphyseal area at the ends of the long bones. Undue force can easily produce a fracture at this site. All manipulations should be performed in such a way that traction is employed to separate the joint surfaces rather than that one bone is levered on another, so compressing the articular cartilage.

(h) Certain joints lend themselves well to manipulation, especially cervical intervertebral joints, shoulder joints and tarsal and metatarsal joints of the feet.

(i) All manipulations should be followed by repeated active exercises carried out by the patient with the aid of analgesics and mechanical aids to movements, such as sling suspension using springs or weights and pulleys, or immersion in saline or mineral baths.

\section{Reconstructive surgery}

\section{(a) Arthrodesis}

As ankylosis often is the natural end result in a joint affected with rheumatoid arthritis, formal arthrodesis does not often have to be performed.' When it appears from the X-ray and clinical appearances in a case that ankylosis of a joint or joints is inevitable, then care should be taken by adequate splintage that ankylosis occurs in the position of optimum function. Should ankylosis threaten to take place in a joint where movement is essential, then it is often better to allow ankylosis to occur and later to do a formal arthroplasty, than to distress the patient unnecessarily by forcing painful movement in a joint where the surfaces have become eroded, the ligaments destroyed by granulation tissues and persisted attempts at movement only serve to stir up a local reaction and keep the tissues hot and painful.

Technique :-

(a) Fixation in plaster in the optimum position. This procedure alone is often sufficient to secure bony ankylosis in a joint if the case is one in which a natural tendency to ankylosis exists. This is true to such an extent that in applying rest plasters in a case of acute rheumatoid arthritis the plasters should always be bivalved within 24 to 48 hours to allow one period of movement daily in every case.

(b) Fixation in plaster combined with bone drilling. A small incision is made down to the bone at a convenient spot near one side of the joint, and by means of a hand drill numerous small channels are drilled across the joint line. This procedure is comparatively 
rapid and atraumatic, and is very helpful in cases where a painful, unsound ankylosis persists in spite of rest and plaster fixation.

(c) Transfixion of the joint surfaces by a steel pin or screw or by a bone peg or graft. This can often be done through a small incision if desired through a window in the plaster and can be combined with bone drilling. It has the added advantage that the post-operative immobilization is complete and consequently the relief of pain on movement is immediate and ankylosis occurs more rapidly.

(d) In cases where there is great proliferation of the synovial membrane or where the joint capsule is distended by a large persistent effusion into the synovial sac, then a formal arthrodesis may be required. This is carried out in a bloodless field under a tourniquet where possible. The whole of the synovial sac is excised, the degenerate articular cartilage is removed from the bone ends, and the raw surfaces carefully fitted together in the position of optimum function. It is better to maintain this position by internal fixation as well as external splintage owing to the risk of slipping in a padded post-operative plaster. Bone grafts driven across the joint surface are best, but stainless steel or Vitallium pins or screws may be employed instead where it is not considered desirable to prolong the operation by cutting a bone graft or where metallic substances give a better initial fixation.

(e) The use of cancellous bone grafts is not often indicated in rheumatoid arthritis owing to the natural tendency to ankylosis being strong, but they are sometimes useful to fill in gaps in irregular bone surfaces and so avoid undue shortening.

Optimum position for arthrodesis. This may or may not coincide with the optimum position for rest in plaster. For instance, in the case of the knee joint, the position for rest in plaster is just short of full extension, while the position for arthrodesis is in about $30^{\circ}$ of flexion; so as to allow the patient to sit down in comfort.

\section{Usual position for arthrodesis :-}

I. Thumb-in position of abduction and opposition to fingers, as in grasping a glass.
2. Wrist-in slight dorsiflexion.

3. Elbow-at $120^{\circ}$ and semi-pronated, but some cases may prefer more flexion.

4. Shoulder $-45^{\circ}$ abduction, $45^{\circ}$ forward flexion and $15^{\circ}$ external rotation.

5. Hip-at about $25^{\circ}$ flexion.

6. Knee-at about $150^{\circ}$.

7. Ankle-in slight plantar flexion, i.e. about $95^{\circ}$ to $100^{\circ}$.

Individual variation in position for arthrodesis. Each case must be considered on its merits, and the patient's occupation, age, habits and the position of neighbouring joints must all be considered.

In the case of a patient who is compelled to spend most of the time sitting down, the knee and hip joints may be arthrodesed in a greater degree of flexion than would otherwise be the case. If one knee is already ankylosed in some flexion, then the second knee, if it has to be arthrodesed, should be done to match, and so on. If both elbows are ankylosed then it is more convenient to have one semi-flexed and the other semi-extended.

\section{(b) Arthroplasty}

This operation must be approached with caution in cases of rheumatoid arthritis owing to the strong tendency to ankylosis present in this disease. A successful arthroplasty demands for its success several factors :-

I. Perseverance on the part of the patient to enable him to endure the arthroplasty in spite of pain.

2. Sufficient muscular power in the affected limb to provide good active control over the new joint.

3. The disease must be arrested or at least in a quiescent phase. Local quiescence of the disease often means bony ankylosis and it is a fact that the most successful arthroplasties are performed in joints where solid bony ankylosis has supervened.

4. The new joint must be protected by splintage for some months after operation in order to enable some degree of stability to be achieved or a useless, flail joint will result.

From this it follows that the indications for arthroplasty in a case of rheumatoid arthritis are limited, but within these narrow limits the operation is of value in selected cases. 
Joints most suitable for arthroplasty :-

(a) Tempors-mandibular joint of jaw.

(b) Acromio-clavicular joint.

(c) Elbow joint.

(d) Inferior radio-ulnar joint.

(e) Metacarpo-phalangeal joints of the thumb and fingers.

(f) Hip joints.

(g) Metacarpo-phalangeal joint of the great toe.

Technique of arthroplasty. The modern tendency is for simplification of technique and the essentials are :-

I. Removal of sufficient bone to allow free movement at the false joint.

2. Prevention of subsequent ankylosis by post-operation traction. This may be achieved in such joints as the temporo-mandibular joint, and the elbow joint, by the aid of gravity alone and the inferior radio-u'nar joint by the fact that the severed bones are held apart by the radius, but in most joints continuous traction, either by skeletal transfixion or by skin traction is maintained for some weeks after the operation.

3. The inter-position of substances between the bony ends. Fascia lata is often employed with success, especially in the elbow joint, but some surgeons hold its use to be unnecessary if continuous post-operative traction is employed.

4. The use of metallic cups between the bone ends, such as the Vitallium cup employed largely in arthroplasty of the hip. The results from this procedure have often been disappointing in cases of rheumatoid disease, although very promising results have been obtained by many surgeons in the treatment of osteoarthritis of the hip by this means.

5. Early movement of the newly-formed joint. Passive movements often have to be employed at first until the inhibiting action of pain has been overcome. Great care must be taken that passive movements are not undertaken too vigorously or too often or an excessive reaction will be set up and ankylosis will be the result.
6. Active exercises. These are the keynote of success. The patient must re-educate his own muscles to move the new articulation and muscles long weakened by atrophy of disuse and by inflammatory fibrosis must be redeveloped by all possible means.

7. Physiotherapy, especially by supervised movements with the limb suspended in slings, or by immersion in warm water, is of great value at this stage. Local heat, gentle massage, mud packs, warm baths, etc., are helpful in relieving pain, promoting comfort and encouraging movement by helping muscular development and improving circulation.

Pseudarthrosis. There are certain sites where a pseudarthrosis is to be preferred to a formal arthroplasty, either because of the local anatomy or because of the strong tendency to ankylosis following any procedure.

At the temporo-mandibular joint, the acromio-clavicular joint, the sterno-clavicular joint and the inferior radio-ulnar joint a simple removal of sufficient bone to leave a wide gap at the site where the false joint is intended is usually better than any formal attempt to fashion a new joint.

In cases where movement is desired at the hip in a case of spondylitis ankylopoetica, then a pseudarthrosis is nearly always preferable to any arthroplasty as the natural tendency for a secondary ankylosis is very strong.

If, however, the head and neck of the femur is removed and traction kept on the femur for six weeks so that a wide gap is left between the bony surfaces, then a free joint may be expected to form.

Mr. Stamm has successfully combined this procedure with a trochanteric osteotomy so as to secure more stability, as in the Schanz osteotomy for congenital dislocation of the hip, and thus obviating the need for wearing a caliper splint for walking. In the simple pseudarthrosis of the hip, however, the wearing of a caliper is essential to providè stability when the patient walks and this should be hinged at the knee to allow the patient to sit in comfort. 